\title{
A CUMULATIVE STUDY OF PERFORMANCE DEGRADING FACTORS IN A HIGH CAPACITY CELLULAR ZONE SYSTEM
}

\author{
RAJEEV KUMAR MITAL \\ Dept. of Electronics \& Communication Engineering, Delhi Institute of Technology \\ Delhi (India) \\ UMESH KUMAR \\ Senior Member, IEEE: Fellow, IETE. Department of Electrical Eng., IIT New Delhi \\ pin-110016 India.
}

\begin{abstract}
In high capacity cellular zone systems, cell size is reduced considerably: hence cells may be re-defined as microcells. The key point in microcell interference modeling is that the desired signal, as well as co-channel interferers' signals should have different statistics. A microcell interference model that which provides different fading parameters to the concerned signals is used in this paper to evaluate various performance degrading factors. Outage probability and spectrum efficiency have been calculated. Various techniques to counteract the above mentioned interferences have been suggested.
\end{abstract}

\section{INTRODUCTION}

The cellular zone system concept has been recognized as the most promising approach to the mobile communications of the future. Within dwindling frequency reserves, it is one of the most practical solutions to the problem of steadily increasingly demand for land mobile radio services. The service quality provided is comparable to Public Service Telephone Networks with additional advantages of spectrum economy, flexible growth, and low power requirements (Fig. 1).

The principle of cellular system is space division multiplexing. A large service area is divided into cells with diameter $(2-20 \mathrm{~km})$, each of which has a number of RF channels assigned, and low power transmitter with the coverage area equal to the cell area. Since the coverage area is small, the same set of frequencies can be used at distances sufficiently apart from the cell where it is being presently used. Coordination among different cells is provide by the Mobile Telephone Switching Office. Fig (2 \& 3)

\section{NAKAGAMI DISTRIBUTION \& $M x / M y$ MODEL}

The Nakagami Distribution is given by:

$$
p \alpha(\alpha)=\frac{2}{\Gamma(m x)}\left(\frac{m x}{2 X m}\right)^{m x} \alpha^{2 m x-1} \exp \left(-\frac{m x}{2 X m} \alpha^{2}\right)
$$

Assistant Professor, Department of Electrical Engineering, I.I.T., Hauz Khas, New Delhi-110016, India Phone: (91)-(11)-3263535 (Residence) (91)-(11)-666979/Extn. 2218 (Office) Fax: (91)-(11)-6862037 (Office) 


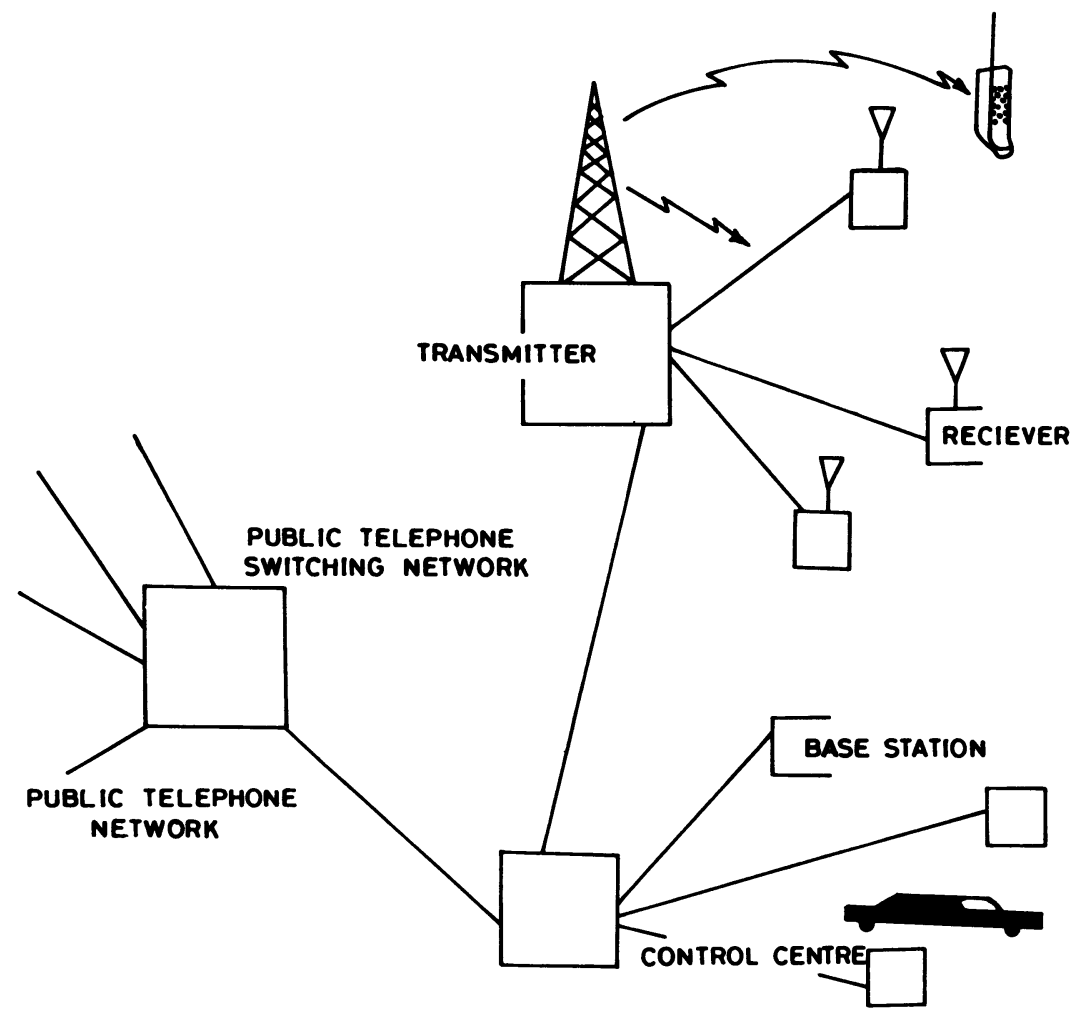

FIGURE 1 A General Cellular System

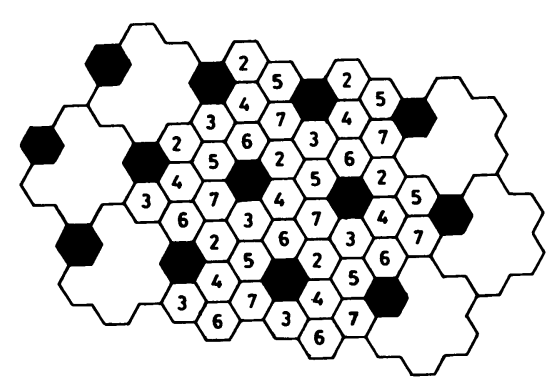

FIGURE 2 A Typical Cell Configuration

where $\Gamma=$ gamma function

$$
\mathrm{Xm}=\text { Mean signal power }
$$

The signal power $x$ measured over one $f$ cycle is

$$
\begin{aligned}
p x(x) & =p \alpha(\alpha) \frac{d \alpha}{d x} \\
& =\frac{1}{\Gamma(m x)}\left(\frac{m x}{X m}\right)^{m x} x^{m x-1} \exp \left(-\frac{m x}{X m} x\right)
\end{aligned}
$$

which is the gamma performance degrading factors (pdf). 


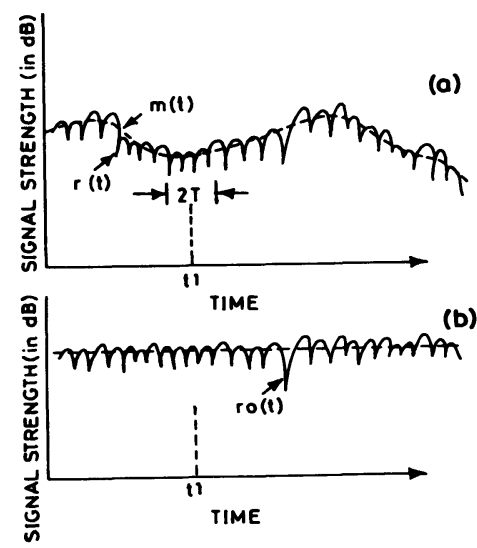

FIGURE 3 (a) A Mobile Radio Signal Fading. (b) A Short Term Signal Fading.

Equations 1 \& 2 degenerates into Rayleigh \& exponential distributions.

The desired and undesired signals have different fading parameters as explained earlier. The desired as well as undesired interfering signals are provided by the same Nakagami characteristics, but with different parameters, i.e., different amounts of fading (A.F) is provided. This model is termed a Nakagami $M x / M y$ model. It relates the A. F. of desired and undesired signals. [1]

The pdf of the undesired signal is calculated by assuming $I$ mutually independent interferers each with A.F.-1/My and mean power $Y_{m}$

$p y(y)=\left(\frac{m y}{Y m}\right)^{m y I} \frac{y^{M y I-1}}{\Gamma(m y I)} \exp \left(-\frac{m y}{Y m} y\right)$

Similarly for the case in which interferers have different mean power, pdf is obtained as follows:

If $I$ th interferer has mean power $Y m i(Y m i-Y m j)$, pdf is obtained as follows

$p y(y)=\sum_{i=1}^{I} Y m_{i}^{I-2} \exp \left(-\frac{y}{Y m i}\right) \prod_{j=1}^{I} \frac{1}{Y m i-y m j}$

\section{CO-CHANNEL INTERFERENCE MICRO CELL INTERFERENCE MODEL}

Co-channel interference occurs when another transmitter on the same carrier frequency as the given transmitter impinges on the receiver. 

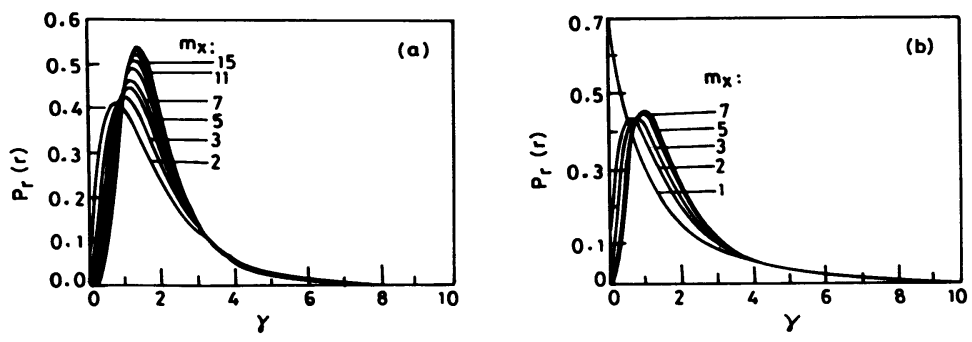

FIGURE 4 (a) p.d.f. of S /I POWER RATIO $M y=$ 2(EQUAL MEAN INTERFERENCE POWER) $(1-3)(B m=5)(b)$ p.d.f. of $S / I$ POWER RATIO $M y=1$ (DIFF. MEAN INTERFERING POWER) $(1=3)[(B m=3) ;(B m=5) ;(B m=7)]$

From the $M x / M y$ model, the pdf of the signal to interference power ratio is calculated as follows:

Assuming the signal to interference power ratio $r=x / y$

$$
\begin{aligned}
p_{r}(r) & =\int_{0}^{\infty} y_{x}(x) p_{y}(y) d y \\
& =\frac{\Gamma(m x+m y I)}{\Gamma(m x) \Gamma(m y I)}\left(\frac{m x}{x m}\right)^{m x}\left(\frac{m y}{y m}\right)^{m y} I
\end{aligned}
$$

This analysis is valid only for the case where co-channel interferers have the same power $y_{m}$.

Similarly for the case in which some interferers have different mean powers, equation 2 is used to calculate pdfs.

$p_{r}(r)=m y^{m x+1} r^{m x-1} \sum_{i=1}^{I} \frac{b m i}{(m x \gamma+b m i)} m x+1 \prod_{j=1}^{I} \frac{b m j}{\prod_{j \neq i}}$

The pdf curves of signal to interferer power ratio are plotted as shown in Fig: 4 Thus, it is quite obvious that frequency re-use is not a straight forward simple procedure.

Another important aspect is that for any given criterion signal level, actual coverage though approximated by hexagonal cells would actually be a blotch (irregular \& indefinite). Truly speaking, isometric contours of co-channel interferers and not the signal power defines cell boundary.

Many good frequency allocation schemes have been developed to reduce co-channel interference, yet it remains the most important system design parameter in cellular system. [2] 


\section{INTERMODULATION AND SENSITIVITY SUPRESSION}

When a mobile station comes close enough to a base station, i.e., visible from a base station antenna, the input to a base station receiver becomes excessive, causing interference.

If there is an excessive input of one channel, the receiver is affected by a sensitivity supressior phenomenon of increasing equivalent noise. However, if there is an excessive input of many channels, the non-linearity of the high frequency circuit in the receiver causes beat interference by intermodulation of these inputs.

Intermodulation may cause interference of the co-channel frequency beat component as well as a malfunction of the squetch circuit, which may make beat channels unusable. Table 1 . shows the various three and five order intermodulation products.

A considerable source in this category is UHF television interference. Interference occurs both ways, i.e., ITV interference to cellular system created by UHF receiver and TVI interference to a UHF TV receiver by a cellular system. A strong signal created by a UHF TV transmitter, operating below the telephone band, will heterodyne with the mobile transmitter producing various order intermodulation products: third-order product being the major component.

2A - B Higher probability

A - B - C

Example:If Ft is the transmitting frequency of the mobile unit.

Fr is the receiving frequency of the mobile unit

Fr is the interfering frequency

$2 \mathrm{Ft}-\mathrm{Fu}=\mathrm{Fr}$

$\mathrm{Fu}=\mathrm{Fr}-90 \mathrm{MHz}$

At a mobile receiver frequency of $870-890 \mathrm{MHz}$ the interferring frequency lies in $780-800 \mathrm{Mhz}$.

Sensitivity supression: Geographical distance, separation often helps in reducing signal interference. However, a mobile radio communications may be hurt by geographical distance separation. The signal received from the mobile unit closer to the base station is stronger than that received from the unit farther away.

TABLE I. NUMBER OF IM PRODUCTS OF VARIOUS ORDERS

\begin{tabular}{|c|c|c|c|}
\hline IM Form & $\begin{array}{c}\text { Order } \\
\text { m }\end{array}$ & $\begin{array}{l}\text { Number of } \\
\text { Frequencies } \\
L \text { in this IM }\end{array}$ & $\begin{array}{l}\text { Total Number of IM } \\
\text { Products of This Form }\end{array}$ \\
\hline $\begin{array}{l}2 \alpha-\beta \\
{ }^{\alpha} \alpha+\beta-\gamma\end{array}$ & $\begin{array}{l}3 \\
3\end{array}$ & $\begin{array}{l}2 \\
3\end{array}$ & $\begin{array}{c}N(N-1) \\
T_{3} \frac{\Delta}{N(N-1)(N-2) / 2} \\
N(N-1)(N-2) / 2\end{array}$ \\
\hline $\begin{array}{l}3 \alpha-\beta-\gamma \\
\alpha+2 \beta-2 \gamma\end{array}$ & $\begin{array}{l}5 \\
5\end{array}$ & $\begin{array}{l}3 \\
3\end{array}$ & $\begin{array}{c}N(N-1)(N-2) \\
T_{5} \frac{\Delta}{N} N(N-1)(N-2) \\
(N-3)(N-4) / 12\end{array}$ \\
\hline${ }^{*} \alpha+\beta+\gamma-\sigma-\eta$ & 5 & 5 & \\
\hline
\end{tabular}

The notation $\alpha, \beta, \gamma, \sigma$ or $\eta$ represents any of the input frequencies but $\alpha-\beta=\gamma-\sigma=\eta$

${ }^{*}$ Indicates the dominant form of the third and fifth-order products. 


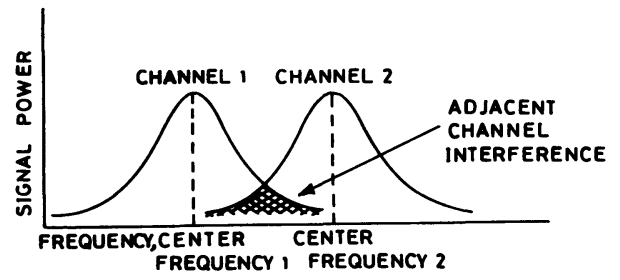

FIGURE 5 ADJACENT CHANNEL INTERFERENCE

\section{ADJACENT CHANNEL INTERFERENCE \& INTER SYMBOL INTERFERENCE}

Adjacent channel interference occurs when energy from one channel spills over to an adjacent channel or filters are loose and capture energy from a broader band than it really needs to (Fig.5)

Intersymbol interference or delay spread is due to relative large delay spread in multipath medium. Since the signal follows different paths, and reflected path tends to be longer than direct paths, different paths of arrival result. The effect is to smear or spread out the signals. This results in symbols overlapping with preceding and following signals. [3]

\section{OUTAGE PROBABILITY \& SPECTRUM EFFICIENCY}

In cellular radio systems, adequate signal strength and adequate signal to interference ratio are essential for communications. Therefore, Outage Probability is defined as the probability of failing to achieve simultaneously a signal-to-noise ratio and signal-to-interference ratio sufficient to give satisfactory reception. It is an important parameter that determines spectrum efficiency. Assuming signal-tointerference protection ratio $\mathrm{Ri}$ and equal mean powers (Fig. 6).

$$
\begin{aligned}
\text { Pout } & =\mathrm{P}_{r}(r<R I) \\
& =\int_{0}^{R_{I}} p_{r}(r) d r \\
& =\frac{\Gamma(m x+m y I)}{\Gamma(m x) \Gamma(m y I)}\left(\frac{m x}{b m}\right)^{m x} m y^{m y} I \int_{0}^{R_{I}} r^{m x-1} . \\
& =\frac{\Gamma(m x+m y I)}{m x \Gamma(m x) \Gamma(m y I)}\left(\frac{m x R I}{m y b m}\right)^{m x} \cdot F\left(m x+m y I m x ; 1+m x ;-\frac{m x}{m y b m} R_{I}\right)
\end{aligned}
$$

$F(\alpha, \beta ; \gamma ; z)=\frac{\Gamma(\gamma)}{\Gamma(\alpha) \Gamma(\beta)} \sum_{n=0}^{\infty} \frac{\Gamma(\alpha+n) T(\beta+n)}{\Gamma(\gamma+n)} \cdot \frac{Z^{n}}{L n}$ 

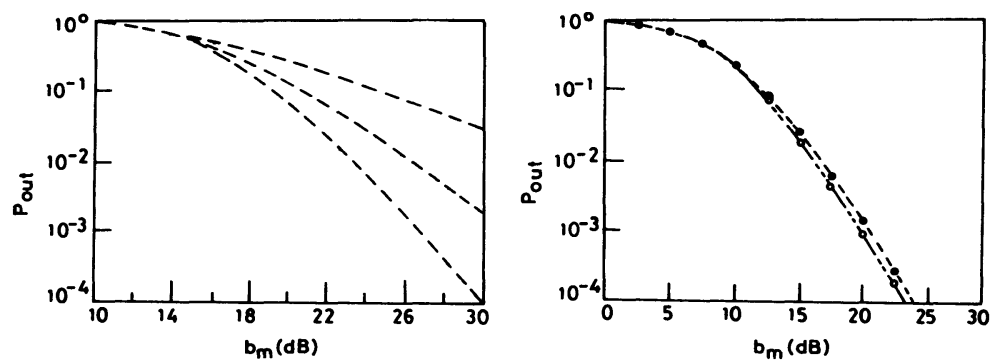

FIGURE 6 OUTAGE PROBABILITY $(R i=5)$

$$
\begin{aligned}
\mathrm{P}_{\mathrm{out}}= & \left(m x R_{I}\right)^{m x} \sum_{i=1}^{I} b m i^{-m x} \times F\left(m x+1, m x ; m x+1 ;-\frac{m x}{b m i} R_{I}\right) \\
& \times \prod_{j=1}^{I} \frac{b m j}{b m j-b m i}
\end{aligned}
$$

Thus the Namagami $M x / M y$ model provides lower outage probabilities in microcell systems compared with medium/large systems. Further, an interesting observation is that one interferer with average power $\mathrm{Ym}$ and two interferers with average power $\mathrm{Ym} / 2$ give almost the same result.

Also, incorporating a signal-to-noise requirement assuming the min. required desired power signal ( $x R n . X m) \& S / I$ Protection ratio $\mathrm{Ri}$. outage probability can be evaluated from the following equation:

$$
\begin{aligned}
\mathbf{P}_{\text {out }} & =1-P_{r}\left(x>R n \times m, \frac{x}{y}>R I\right) \\
& =1-\int_{R n \times m}^{\infty}\left[\int_{0}^{X / R I} p y(y) d y\right] p x(x) d x
\end{aligned}
$$

The performance of a cellular system can be measured in terms of a $G E O$ GRAPHICAL SPECTRUM EFFICIENCY. It relates the average size of the cell to the degree of frequency re-use after accounting for various interferences.

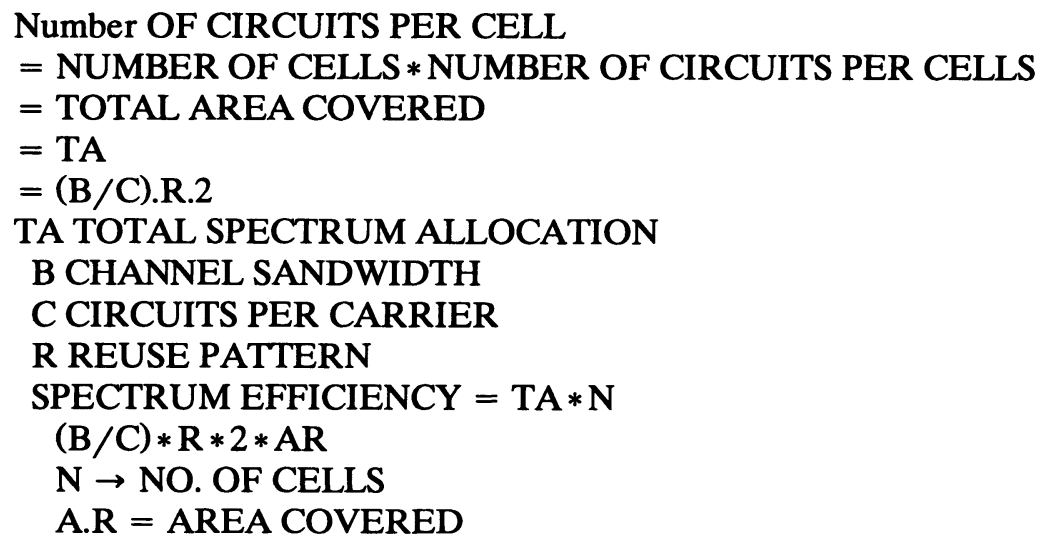


As a rough estimate: for a 1 mile radius cell with 900 cells covering the area and a seven cell re-use pattern, the efficiency turns out to be 15.2 CIRCUIT/MHz/sq.mile.

\section{CELL RE-USE DISTANCE}

It is another important system design parameter. The pdf and outage probability was determined earlier. The relation between outage at all fringes and outage with in cell coverage area may be obtained as follows:

$X_{m}=A \cdot \gamma^{-\alpha}$

Area mean of $\mathrm{CIR}=\left(\frac{R}{D-R}\right)^{-\alpha}=\Lambda m(R)$

$\frac{D}{R}=1+\Lambda m(R)^{1 / \alpha}$

\section{COUNTERMEASURES TO MINIMIZE THE EFFECT OF INTERFERENCE}

The main measures include directive antennas, diversity measures, and supplementary base stations.

\section{1) DIRECTIVE ANTENNAS:}

Here all antennas associated with the same rf channel, will point in the same direction. Referring to the Illustrative fig. 7 mobile unit $Z$ receives full power from $A$, reduced power from $B \& C$, greatly reduced power from $D \& E$, and only back radiation from $F$.

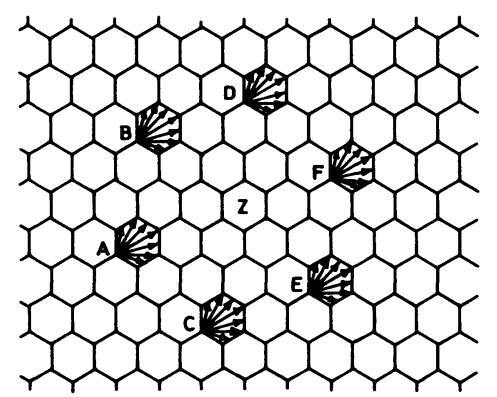

FIGURE 7 Use of directive antennas. 
A considerable reduction in co-channel interference is also achieved. Very often, a tilting antenna configuration is used. A notch is created in the radiation pattern of an antenna, which is very effective in reducing co-channel interference. [4]

\section{2) DIVERSITY MEASURES:}

Refers to any of the several techniques for sampling the received signals more than once and either by combining these signals or selecting the best of them and thus, improving the $\mathrm{S} / \mathrm{N}$ ratio at the receiver.[5]

\section{Space Diversity:}

In layman's term, simply having two or more antennas separated by a minimum of half wavelength. Two antennas, so separated it will show an correlated fading pattern. So if one antenna is in deep fade, the other will not be in fade. So radio circuit may select the best signal.

In more generic terms, a multiple antenna system may be used to reduce short term fading. It significantly removes co-channel interference.

\section{Polarization Diversity:}

The signals transmitted with both horizontal and vertical polarization exhibit uncorrelated fading statistics. So antennas for receiving both type of polarization are installed. It is a special case of space diversity.

\section{Frequency Diversity:}

Involves transmission of the same circuit. On two different frequencies separated such that again fading characteristics are related. This approach is practically useless as it wastes a lot of spectrum.

\section{Time Diversity:}

Means transmitting identical messages at different time slots, which yield two uncorelated signals at the receiver end. It is good for reducing intermodulation at a multichannel site.

\section{3) SUPPLEMENTARY BASE STATIONS}

To fill in large holes created by shadowing from hills or buildings, the answer has been to deploy corner sited antennas with directional transmission to provide an alternate path for signals (Fig.13). But due to the fuzziness of cell boundary, a temporary fade results in hand-off. So, the number of hands-off increase

Finally any of these techniques may be used alone or as a combination of these techniques. 
TABLE 2. FREQUENCY PLANS TO AVOID ORDER 3 IM PRODUCTS.

\begin{tabular}{lccl}
\hline $\begin{array}{l}\text { Signal } \\
\text { Channels }\end{array}$ & N & $\begin{array}{c}\text { Total } \\
\text { Channels } \\
\mathbf{M}\end{array}$ & Frequencies $\boldsymbol{F}_{\boldsymbol{i}}$ \\
\hline IM & 3 & 4 & $1,2,4$ \\
product & 4 & 7 & $1,2,5,7$ \\
spreading & 5 & 12 & $1,2,5,10,12$ \\
& 6 & 18 & $1,2,5,11,13,18$ \\
& 7 & 26 & $1,2,5,11,19,24,26$ \\
& 8 & 35 & $1,2,5,10,16,23,33,35$ \\
& 9 & 46 & $1,2,5,14,25,31,39,41,46$ \\
\hline No IM & 10 & 62 & $1,2,8,12,27,46,48,57,60,62$ \\
product & 3 & 7 & $1,3,7$ \\
spreading & 4 & 15 & $1,3,7,15$ \\
\hline
\end{tabular}

\section{4) COUNTERING INTERMODULATION}

Suppose that $N$ frequency sinusoids subchannels are to be packed in a combined channel of bandwidth M.B.

M Total no. of channels required to avoid I.M. among $N$ channels

B Bandwidth of sub channels.

The Table 2 shows the minimum bandwidth to avoid third order \& fifth order intermodulation for a given $N$.

\section{CONCLUSION}

The performance degrading factors in microcellular systems has been investigated in this paper. An interference model named Nakagami $M x / M y$ model is explained and used. The desired signal, as well as co-channel interferers, are assumed to be subjected to Nakagami fading but the co-channel interferers experience deeper fading $(M y / M x)$, which characterizes the microcellular environment. Numerical results show that a lower outage probability is achieved in microcellular systems compared with medium/large cell systems in which the desired signal and interferers usually experiences the same amount of fading. The diversity effect on the microcellular systems has also been studied.

\section{REFERENCES}

1. Richard C. French Effect of Fading and Shadowing on Channel Reuse Distance in Mobile Radio" IEEE Trans Veh. Technol. pp 171-187. 1979

2. Kota Kinoshita Masaharuhata, "Radio link design of cellular land mobile communications." IEEE Trans Veh. Technol pp 25-37 1981

3. Y.D. Yao and A.U.H Sheikh "Investigations into Co-Channel Interference in Microcellular mobile radio systems" IEE Trans. Veh. Technol. pp 114-123 1991

4. W.C.Y. Lee, Mobile Communication Engineering New York: MC Graw Hill 1982

5. "Advanced mobile phone services" special issue, Bell System technical journal Vol. 58 Jan 1979. 

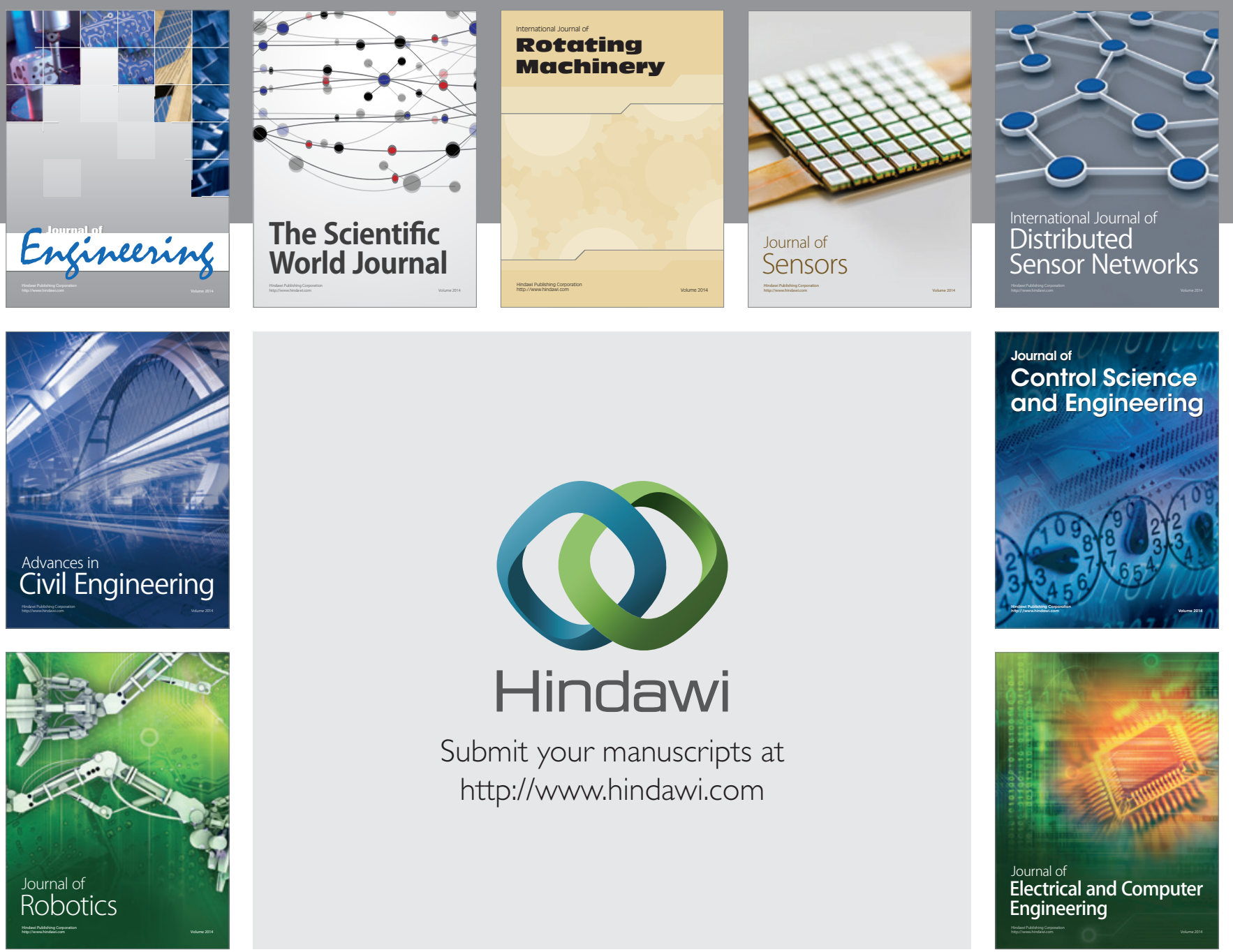

Submit your manuscripts at

http://www.hindawi.com
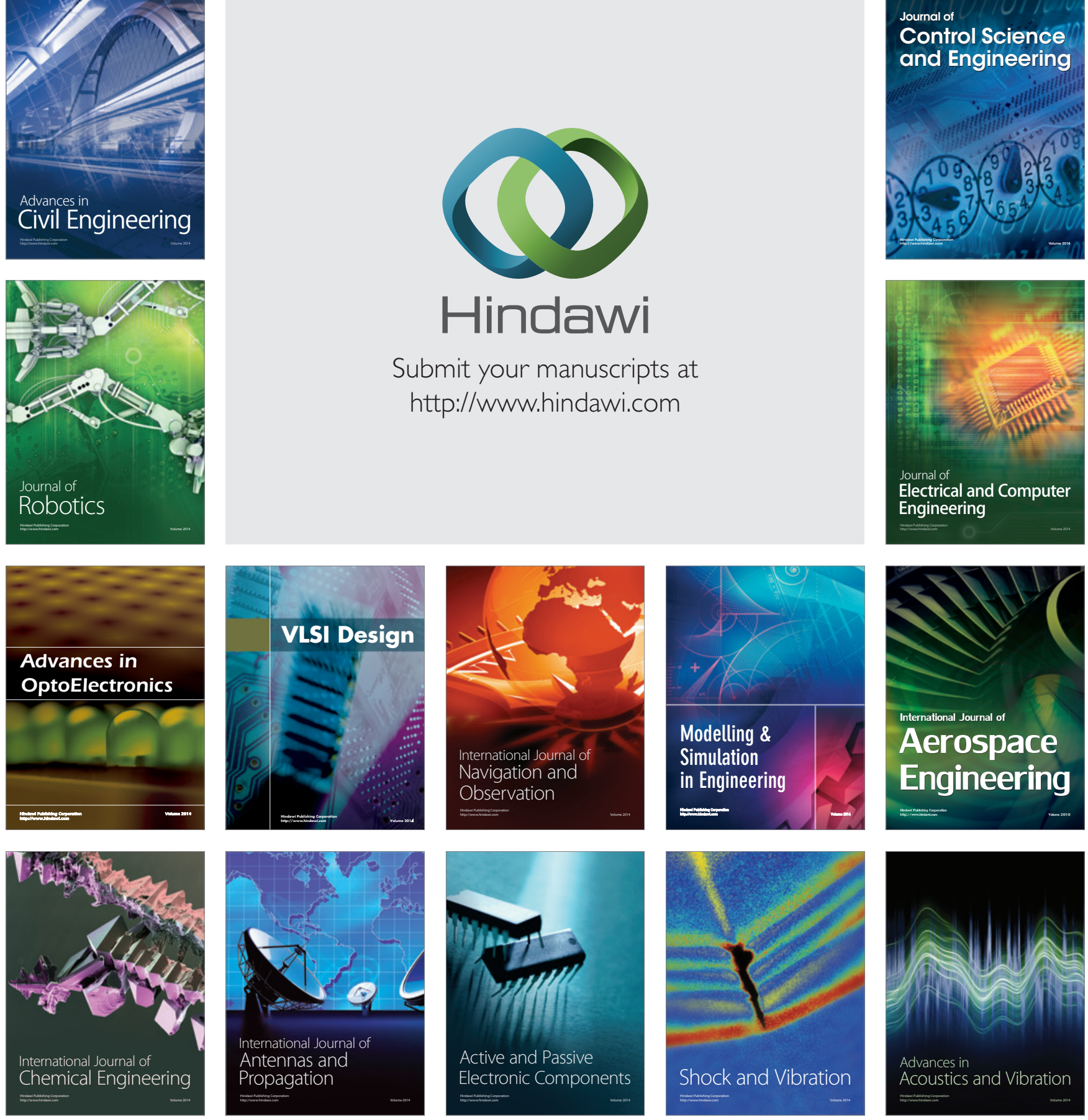